Research Article

\title{
Nonlinear Dynamic Performance of a Bolt-Disc Rotor with the Position Error of Circumferential Bolt-Holes
}

\author{
Haibo Zhang, ${ }^{1}$ Yi Liu $\left(\mathbb{D},{ }^{2}\right.$ and Xin Huang ${ }^{3}$ \\ ${ }^{1}$ Wuhan Institute of Marine Electric Propulsion, Wuhan, China \\ ${ }^{2}$ Key Laboratory of Education Ministry for Modern Design and Rotor-Bearing System, Xi'an Jiaotong University, \\ Xi'an 710049, China \\ ${ }^{3}$ AECC Guiyang Engine DesignResearch Institute, Guiyang, China
}

Correspondence should be addressed to Yi Liu; sfzw0016@163.com

Received 25 December 2020; Revised 2 February 2021; Accepted 3 April 2021; Published 7 May 2021

Academic Editor: Fabio Rizzo

Copyright ( 2021 Haibo Zhang et al. This is an open access article distributed under the Creative Commons Attribution License, which permits unrestricted use, distribution, and reproduction in any medium, provided the original work is properly cited.

\begin{abstract}
The bolt-holes in the assembly discs are designed to limit the circumferential displacement of bolts for the bolt-disc rotor. The position error of circumferential bolt-holes is created in a three-dimensional model of bolt-disc rotor. The distribution of nonuniform stress and deformation is acquired according to finite element approach. Static results demonstrate that the position error of bolt-holes leads to obvious concomitant unbalances including constant mass eccentricity and speed-variant bending under the influence of large tightening force. When these unbalance factors are taken into consideration, dynamic performance such as instability areas and nonlinear motions are analyzed by Newton iterative process and a prediction-correction calculation method. Dynamic results show that rotor flexure enables the systematic stability decreased obviously because of this position error. There is a special phenomenon compared to monobloc rotor that the vibration amplitude proceeds to rise when rotating speed exceeds the critical speed. Moreover, the allowable position error of bolt-holes is obviously smaller than that of monobloc rotor and uneven tightening is a feasible way to reduce adverse effects on the dynamic properties when position error appears. This work proposes a static-dynamic approach to investigate the dynamics of imprecise bolt-disc rotor and establishes the relationship between machining error and dynamic features.
\end{abstract}

\section{Introduction}

Bolt-disc rotor is a sort of typical combined rotor, in which many separated parts such as discs are fastened together by one central bolt or several circumferentially distributed bolts. This kind of rotor structure has wide applications in gas turbines, aero engines, and so on. The bolt-holes in the assembly discs are designed to limit the circumferential displacement of bolts. In order to satisfy the high assembly demand, the bolt-holes are manufactured very precisely before the discs are assembled. For example, the position error of bolt-holes in the 17 assembly discs in an F-class gas turbine rotor (Figure 1) is controlled to be less than $50 \mu \mathrm{m}$ when the distributed diameter of bolts is $980 \mathrm{~mm}$. Why the position precision of bolt-holes in the boltdisc rotor is so high? In this paper, this basic problem will be discussed from the perspective of $3 \mathrm{D}$ nonlinear rotor dynamics.
For traditional rotor-dynamic research studies, rotor models are usually treated as rigid rotor, Jeffcott rotor, 1D finite element rotor (e.g., Timoshenko-beam rotor), and so on [1-5]. These models are very classic and useful to analyze the basic dynamic properties, but detailed features (such as holes and defect) in a rotor cannot be reflected entirely because structure simplification exists in these basic rotor models inevitably. In order to describe the actual influence of machining errors, the bolt-disc rotor model should be established in a $3 \mathrm{D}$ way which can contain the entire structure features of the position error of bolt-holes. For the actual needs of the engineering, $1 \mathrm{D}$ bolt-disc rotor is presented [6], but 3D dynamic effects of the bolt-disc rotor cannot be considered in the combined model. Afterwards, 3D nonlinear rotor-dynamic analysis approach is proposed for acquiring the characteristics of the monobloc rotor [7]. 


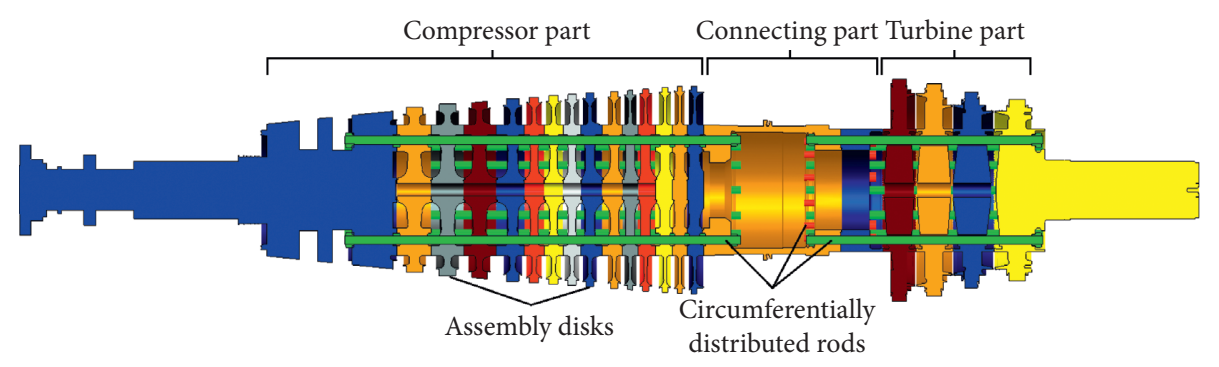

FIgURE 1: Structure of an F-class gas turbine rotor.

However, the position error of bolt-holes causes the mixed static and dynamic problem because uniformly distributed tightening force of bolts can lead to distinct deformation. There is a report [8] that only considers the roughness of interfaces about this static-dynamic uniting problem, but the method is inadequate to analyze the position error because it has larger shape scale and will cause more obvious effects than surface roughness.

In this paper, an actual position error of bolt-holes is considered in a three-dimensional bolt-disc rotor model. The finite element method including contact calculation $[9,10]$ is adopted to analyze the static influences. The consequent 3D rotor-dynamic properties such as stability and vibration are obtained by Newton iterative process, a prediction-correction calculation method, and Floquet theory [11-14]. The comparison between bolt-disc rotor and monobloc rotor is also widely discussed to deduce relative conclusions.

\section{Static Characteristics of an Imprecise Bolt- Disc Rotor with Position Error of Bolt-Holes}

2.1. Structure of Bolt-Disc Rotor Bearing System. Taking the basic structure of compressor in the F-class gas turbine rotor (Figure 1) as reference, an analogous bolt-disc rotor will be studied in Figure 2. This combined rotor has three separated discs ("a," "b," and "c") which are fastened by some circumferential distributed bolts which go through the holes in discs (green lines in Figure 2 and being called bolt-holes). Every bolt has the same size and elongation which is mounted to tighten each rotating disc of this rotor.

During an assembly procedure, the bolt-disc rotor is combined vertically for avoiding the gravity influence. The axial assembly interfaces (blue lines) between three discs are used to ensure sufficient contact stiffness. The concentricity between discs is guaranteed by some coaxial-locating interfaces (red lines). Bolt surfaces are designed to fit against the corresponding disc-hole side faces.

After the above regulated assembly process, the bolt-disc rotor is placed horizontally and supported by 2 cylindrical sliding bearings (" $j$ " and " $k$ "). The rotating speed ranges from 0 to $7500 \mathrm{rpm}$ (working speed). Table 1 shows the variables of bolt-disc rotor and bearing.

2.2. Position Error of Bolt-Holes. The position error of circumferential bolt-holes in this paper is shown in Figure 3(a). The center of the circumferentially distributed bolt-holes on the disc " $b$ " shifts from the $O$ (ideal position) to $O$ ' (actual position) because of this positional error. The deviation $h=1$ OO' is set to be $50 \mu \mathrm{m}$ which compared to the F-class gas turbine rotor. Meanwhile, other two discs are manufactured perfectly.

The 3D imprecise disc and corresponding bolt-disc rotor are discretized by $m$-node $(m=8)$ elements by applying the FE method which are shown in Figure 3(b).

2.3. Static Analysis. The key point of static analysis is to calculate stress and status of contact surfaces in the bolt-disc rotor that are obtained by the iteration [10]:

$$
\left(\begin{array}{cc}
\mathbf{K}^{R}+\mathbf{K}_{\Lambda} & \mathbf{H}^{T} \\
\mathbf{H} & 0
\end{array}\right)\left(\begin{array}{l}
\mathbf{u} \\
\eta
\end{array}\right)=\left(\begin{array}{c}
\mathbf{F}-\mathbf{F}_{\Lambda} \\
-\mathbf{h}_{0}
\end{array}\right)
$$

where $\mathbf{K}^{R}$ is the stiffness matrix of imprecise bolt-disc rotor; $\mathbf{u}$ is displacement vector; $\eta$ is Lagrangian multiplier; $\mathbf{h}$ is contact constraints; $\mathbf{F}$ is force vector; $\Lambda$ is penalty factor; and $\mathbf{K}_{\Lambda}=\mathbf{H}^{T} \Lambda \mathbf{H}$ and $\mathbf{F}_{\Lambda}=\mathbf{H}^{T} \Lambda \mathbf{h}_{0}$ in which $\mathbf{H}=\partial \mathbf{h} / \partial \mathbf{u}$.

Since the constraint conditions change constantly with contact state, the incremental iteration is used to solve the above equations.

For comparison, static analysis is carried out to investigate the relative characteristics of the perfect and imprecise bolt-disc rotor. In Figure 4, at a constant speed (7500 rpm), results indicate that the stress of a bolt (No. 4 bolt, for example) in the perfect bolt-disc rotor is distributed uniformly. However, obviously uneven stress appears on the corresponding bolt in the imprecise bolt-disc rotor.

Due to the existence of the position error of bolt-holes, the bolt-hole surfaces of disc " $b$ " press several bolts when the disc shifts transversely after assembly. As a result, inhomogeneous stress and bending deformation are caused by the pressing force.

Consequently, the position error of bolt-holes leads to mass eccentricity $e$; nonuniform stress triggers axis-center shift $s_{\mathrm{d}}$. Compared to a precise-machining rotor, the position error on disc "b" causes a constant $e$ (equals to $2.98 \mu \mathrm{m}$ ) during the whole procedure and a speed-variant $s_{d}$ in the working procedure which increases gradually up to $6.28 \mu \mathrm{m}$ at working speed $(7500 \mathrm{rpm})$.

On the other hand, $s_{d}$ and $e$ of precise disc are extremely close to zero. It proves that calculating accuracy is absolutely adequate to analyze the static influences of position error of bolt-holes in the rotor. 


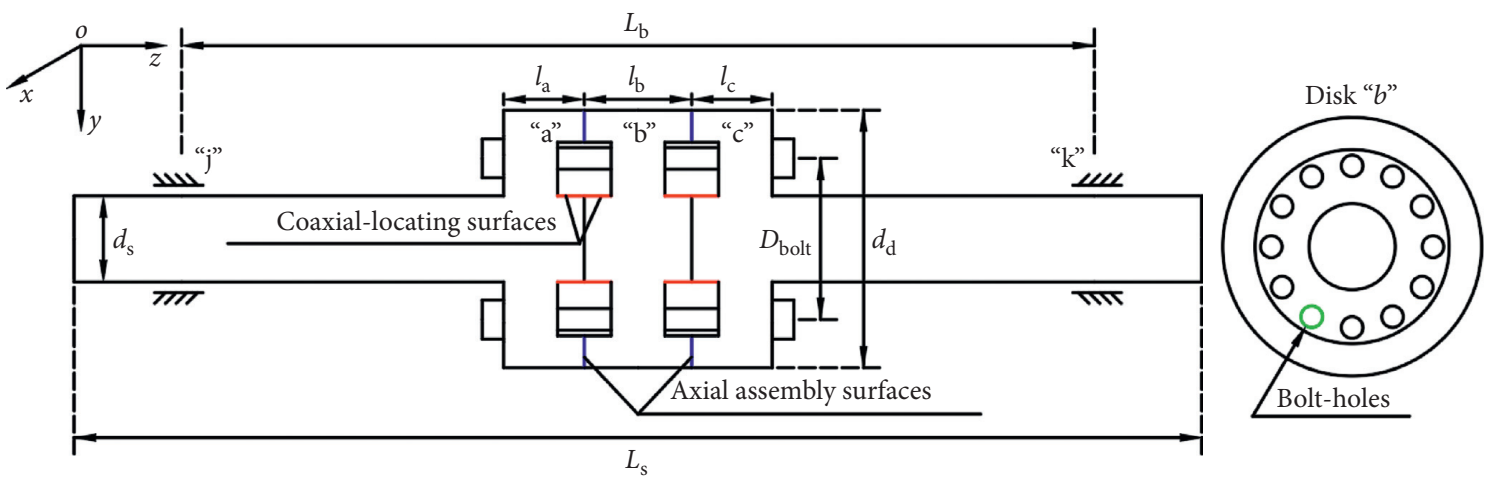

FIgURE 2: Typical bolt-disc rotor bearing system.

TABLE 1: Variables of the bolt-disc rotor bearing system.

\begin{tabular}{lcc}
\hline Item & Variable & Value \\
\hline & Diameter of shaft, $d_{\mathrm{s}}$ & $0.08 \mathrm{~m}$ \\
& Length of shaft, $L_{\mathrm{s}}$ & $1.25 \mathrm{~m}$ \\
& Diameter of discs, $d_{\mathrm{d}}$ & $0.24 \mathrm{~m}$ \\
Bolt-disc rotor & Thickness of disc "a," $l_{\mathrm{a}}$ & $0.075 \mathrm{~m}$ \\
& Thickness of disc "b," $l_{\mathrm{b}}$ & $0.1 \mathrm{~m}$ \\
& Thickness of disc "c," $l_{\mathrm{c}}$ & $0.075 \mathrm{~m}$ \\
& Number of bolts, $n$ & 12 \\
& Tightening elongation, $l_{\mathrm{F}}$ & $0.75 \mathrm{~mm}$ \\
& Diameter of bolts, $d_{\mathrm{b}}$ olt & $0.02 \mathrm{~m}$ \\
& Diameter of circumference for bolts, $D_{\text {bolt }}$ & $0.156 \mathrm{~m}$ \\
\hline Journal bearings & Span of bearing, $L_{\mathrm{b}}$ & $1.05 \mathrm{~m}$ \\
& Radius, $R$ & $0.04 \mathrm{~m}$ \\
& Width, $B_{j}$ & $0.08 \mathrm{~m}$ \\
& Radial clearance, $c_{0}$ & $0.0002 \mathrm{~m}$ \\
& Lubricant viscosity, $\mu$ & $0.0182 \mathrm{Pas}$ \\
\hline
\end{tabular}

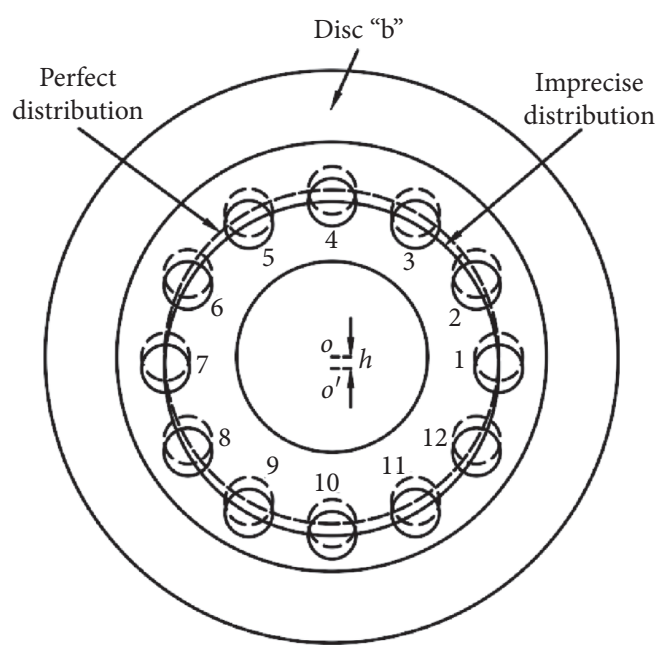

(a)

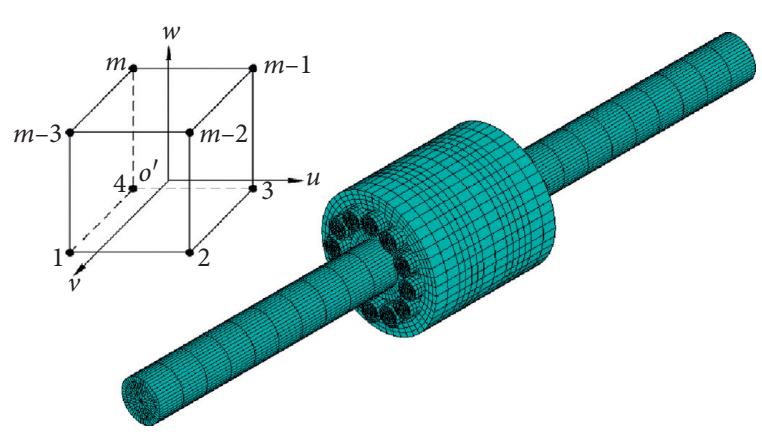

(b)

FIGURE 3: 3D model of bolt-disc rotor with the position error of bolt-holes. (a) Imprecise disc "b" and (b) imprecise bolt-disc rotor.

Then disc-center shift $s_{d}$ enables the combined rotor to have an entire bending $\mathbf{r}_{d}$ whose vector is defined as $\mathbf{r}_{d}=\left(s_{1}, \ldots, s_{d}, \ldots\right.$, $s_{k}$ ) in which $k$ is the node number on the axis (Figure 5). The way to obtaining $s_{k}$ is similar with calculating $s_{d}$. Rotor bending occurs in assembly procedure, and it has been enhanced in working procedure due to centrifugal pull. The bending variation does not absolutely exist in traditional monobloc rotor which does not have circumferential bolts and tightening force. 


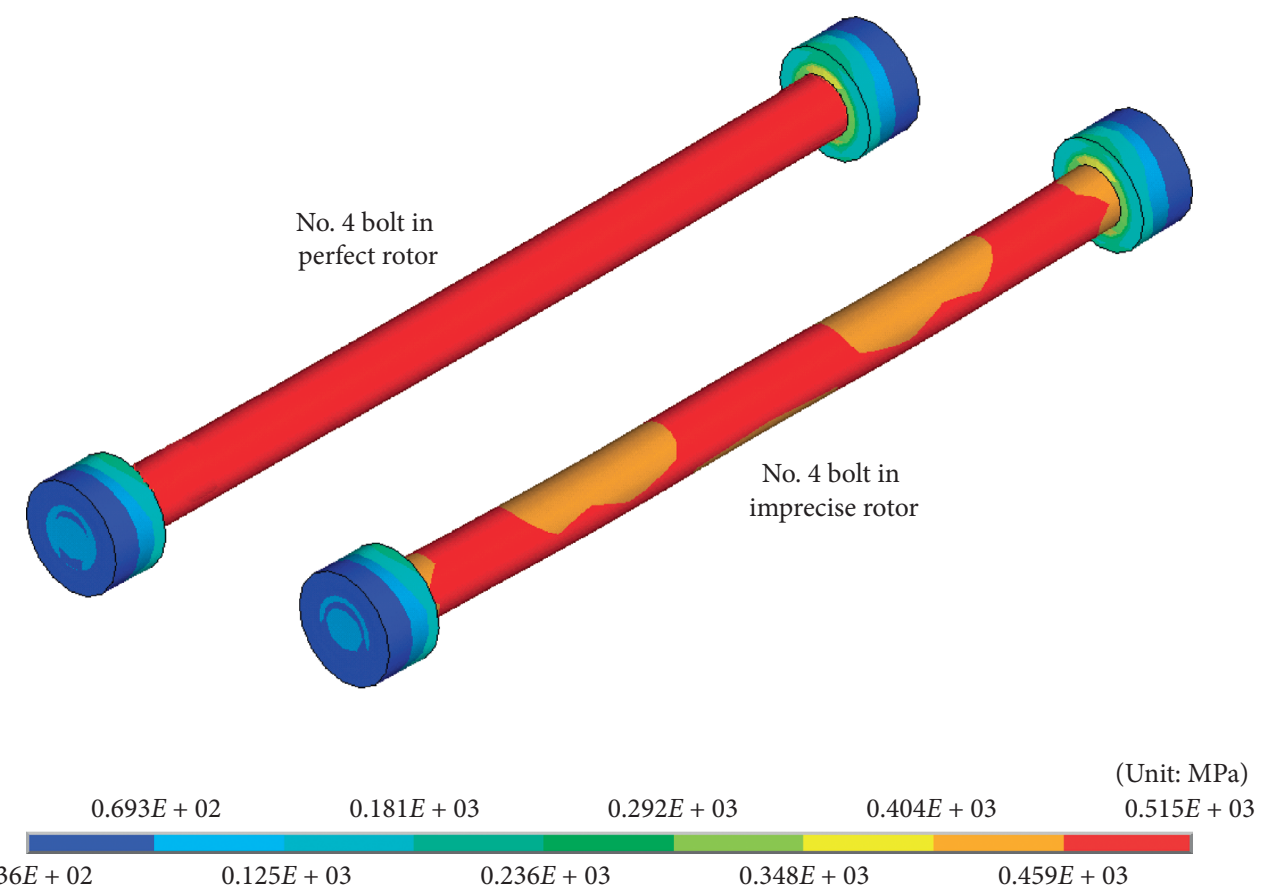

Figure 4: Stress distribution of No.4 bolt in both perfect and imprecise bolt-disc rotor.

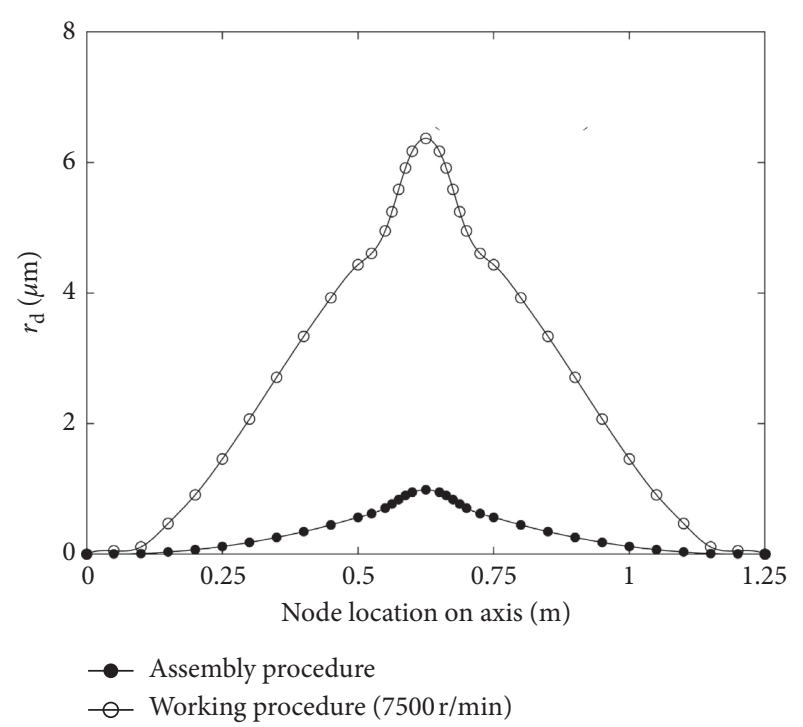

FIGURE 5: Rotor bending of imprecise bolt-disc rotor.

\section{Dynamic Equations and Nonlinear Solution Method}

3.1. Equations of the 3D Imprecise Rotor and System Reduction. When the bolt-disc rotor has the unbalance factor including mass eccentricity and rotor bending, the dynamic model of imprecise bolt-disc rotor system should take them into account.

For the 3D bolt-disc rotor system, the DOFs are divided into nonlinear and linear component. DOFs belong to the bearing nodes (used to add bearing forces) and the disc nodes (used to add unbalance forces) are defined as nonlinear component; all the other DOFs belong to other nodes of the rotor are defined as linear component.

For the nonlinear component, eigenmodes can reflect the dynamic characteristics of the linear system. As a result, the eigenvalues of the equations in the linear component are calculated and the first several eigenmodes are reserved.

Then, dynamic equations are divided into linear and nonlinear sections:

$$
\begin{aligned}
& \left(\begin{array}{cc}
\mathbf{M}_{\mathrm{aa}}^{R} & \mathbf{M}_{\mathrm{ai}}^{R} \\
\mathbf{M}_{\mathrm{ia}}^{R} & \mathbf{M}_{\mathrm{ii}}^{R}
\end{array}\right)\left(\ddot{u}_{a} \ddot{u}_{i}\right)+\left(\begin{array}{cc}
\mathbf{G}_{\mathrm{aa}}^{R} & \mathbf{G}_{\mathrm{ai}}^{R} \\
\mathbf{G}_{\mathrm{ia}}^{R} & \mathbf{G}_{\mathrm{ii}}^{R}
\end{array}\right)\left(\begin{array}{c}
\dot{u}_{a} \\
\dot{u}_{i}
\end{array}\right)+\left(\begin{array}{cc}
\mathbf{K}_{\mathrm{aa}}^{R} & \mathbf{K}_{\mathrm{ai}}^{R} \\
\mathbf{K}_{\mathrm{ia}}^{R} & \mathbf{K}_{\mathrm{ii}}^{R}
\end{array}\right) \\
& \cdot\left[\left(\begin{array}{c}
\mathbf{u}_{a} \\
\mathbf{u}_{i}
\end{array}\right)-\left(\begin{array}{c}
\mathbf{r}_{\mathrm{d} a} \\
\mathbf{r}_{\mathrm{d} i}
\end{array}\right)\right] \\
& =m \omega^{2}\left(\begin{array}{c}
\mathbf{e} \\
0
\end{array}\right)+\left(\begin{array}{c}
\mathbf{g}_{a} \\
\mathbf{g}_{i}
\end{array}\right)+\left(\begin{array}{c}
\mathbf{f}_{B} \\
0
\end{array}\right),
\end{aligned}
$$

where $\mathbf{M}^{R}$ and $\mathbf{G}^{R}$ are mass matrix and gyroscope matrix of rotor; $\mathbf{g}$ is gravity force vector; subscripts " $a$ " and " $\mathrm{i}$ " stand for nonlinear and linear DOFs respectively; and $\mathbf{f}_{B}$ is vector of bearing forces which is calculated based on finite difference method [15]. The pad of cylinder bearing is discretized into many nodes in geometry. The total bearing force is regarded as the sum of hydrodynamic pressure which is solved by Reynolds equation.

In addition, linear DOFs of dynamic equation (2) are reduced by a transformation matrix $\mathbf{T}$ by applying a component mode synthesis method [16]. As a result, the residual equations of imprecise rotor system are listed as 


$$
\mathbf{M}_{0} \ddot{q}+\mathbf{G}_{0} \dot{q}+\mathbf{K}_{0} \mathbf{q}=\mathbf{Q}_{0},
$$

with $\left\{\begin{array}{l}\mathbf{q}=\mathbf{T} \mathbf{u}, \mathbf{Q}_{0}=\mathbf{T}^{T}\left(\mathbf{K}^{R} \mathbf{r}_{d}+m e \omega^{2}+\mathbf{g}+\mathbf{f}_{B}\right) \\ \mathbf{M}_{0}=\mathbf{T}^{T} \mathbf{M}^{R} \mathbf{T}, \mathbf{G}_{0}=\mathbf{T}^{T} \mathbf{G}^{R} \mathbf{T}, \mathbf{K}_{0}=\mathbf{T}^{T} \mathbf{K}^{R} \mathbf{T}\end{array}\right.$.

For the reduced bolt-disc rotor system originated from Figure 3(b), the reserved DOFs equal 27 which includes 3 disc nodes ( 9 DOFs), 2 bearing nodes (6 DOFs), and first 12 order eigenmodes ( 6 rigid eigenmodes and 6 elastic eigenmodes).

3.2. Nonlinear Solution Method. In order to obtain displacement and velocity, state parameter $\mathbf{Y}$ is introduced:

$$
\frac{\mathrm{d} \mathbf{Y}}{\mathrm{d} t}=\frac{\mathrm{d}}{\mathrm{d} t}\left(\begin{array}{c}
\mathbf{q} \\
\dot{q}
\end{array}\right)=\mathbf{F}(t, \lambda, \mathbf{Y}),
$$

where $\mathbf{F}(t, \lambda, \mathbf{Y})=\left(\dot{q} / \mathbf{M}_{0}^{-1}\left(\mathbf{Q}_{0}-\mathbf{K}_{0} \mathbf{q}-\mathbf{G}_{0} \dot{q}\right)\right)$ and $\lambda$ is the excitation variable such as $\omega, e$, and $\mathbf{r}_{d}$.

Since $e$ and $\mathbf{r}_{d}$ meet the periodic feature when rotor rotating, the solutions of equation (4) are determined by

$$
\left\{\begin{array}{l}
\frac{\mathrm{d} \mathbf{Y}}{\mathrm{d} t}=\mathbf{f}(\mathbf{Y}, t, \lambda) \\
\mathbf{Y}(t)=\mathbf{Y}(t+T)
\end{array},\right.
$$

where $T$ is the rotating period.

When $\lambda=\lambda_{0}$, equation (4) can be changed into solving target periodic solutions $\mathbf{Y}(t)$ according to nonlinear definition

$$
\mathbf{H}\left(\lambda_{0}, \mathbf{Y}\right)=\mathbf{Y}(t+T)-\mathbf{Y}(t)=0
$$

Here, the Newton iteration method is applied to approach $\mathbf{Y}(t) . \mathbf{H}\left(\lambda_{0}, \mathbf{Y}\right)$ is determined by initial value $\mathbf{Y}_{0}$ and Jacobian matrix $\partial \mathbf{H} / \partial \mathbf{Y}$ after Taylor expansion:

$$
\mathbf{H}\left(\lambda_{0}, \mathbf{Y}\right)=\mathbf{H}\left(\lambda_{0}, \mathbf{Y}_{0}\right)+\frac{\partial \mathbf{H}}{\partial \mathbf{Y}}\left(\mathbf{Y}-\mathbf{Y}_{0}\right)
$$

After bringing equation (7) into (6), $\mathbf{Y}$ is calculated according to the next Newton iterative:

$$
\mathbf{Y}=\mathbf{Y}_{0}+\left(\frac{\partial \mathbf{H}}{\partial \mathbf{Y}_{0}}\right)^{-1}\left[\mathbf{H}\left(\lambda_{0}, \mathbf{Y}\right)-\mathbf{H}\left(\lambda_{0}, \mathbf{Y}_{0}\right)\right]
$$

where $\partial \mathbf{H} / \partial \mathbf{Y}_{0}$ is obtained according to equation (8) by integrating $T$ :

$$
\frac{\mathrm{d}}{\mathrm{d} t}\left(\frac{\partial \mathbf{Y}}{\partial \mathbf{Y}_{0}}\right)=F_{\mathbf{X}}^{\prime}\left(t, \lambda_{0}, \mathbf{Y}\right) \cdot \frac{\partial \mathbf{Y}}{\partial \mathbf{Y}_{0}} .
$$

When $\lambda$ alters, equation (6) is replaced by $\mathbf{H}(\lambda, \mathbf{Y})=\mathbf{0}$. If $n$ substep solution $\mathbf{Y}_{n}$ is solved, the $n+1$ substep begins with the prediction mode

$$
\left\{\begin{array}{l}
\mathbf{Y}_{n+1}=\mathbf{Y}_{n}-\left[\frac{\partial \mathbf{H}(\lambda, \mathbf{Y})}{\partial \mathbf{Y}_{n}}\right]^{-1} \cdot \frac{\partial \mathbf{H}(\lambda, \mathbf{Y})}{\partial \lambda} \cdot \Delta \lambda \\
\lambda_{n+1}=\lambda_{n}+\Delta \lambda
\end{array}\right.
$$

where $\partial \mathbf{H}(\lambda, \mathbf{Y}) / \partial \lambda$ is solved by the integration of equation (11):

$$
\frac{\mathrm{d}}{\mathrm{d} t}\left(\frac{\partial \mathbf{Y}}{\partial \lambda}\right)=F_{\mathbf{X}}^{\prime}(t, \lambda, \mathbf{Y}) \cdot \frac{\partial \mathbf{Y}}{\partial \lambda}+\mathbf{F}_{\lambda}^{\prime}(t, \lambda, \mathbf{Y})
$$

with $(\partial \mathbf{Y} / \partial \lambda)_{t 0}=\mathbf{0}$ and $(\partial \mathbf{Y} / \partial \lambda)_{(t 0+T)}=\partial \mathbf{H}(\lambda, \mathbf{Y}) / \partial \lambda$.

It ends with the following correction iteration which is based on equation (8) to solve $\mathbf{Y}_{n+1}$ :

$$
\mathbf{Y}_{n+1}=\mathbf{Y}_{n}+\left(\frac{\partial \mathbf{H}}{\partial \mathbf{Y}_{n}}\right)^{-1}\left(\mathbf{H}_{n+1}-\mathbf{H}_{n}\right)
$$

\section{Dynamic Characteristics of Imprecise Bolt- Disc Rotor System}

As a comparison, a same-sized monobloc rotor is analyzed simultaneously. The monobloc rotor's radial-distributed bolts have no elongation, and all the discs are boned together. The monobloc rotor has the same mass eccentricity with the bolt-disc rotor, but it has no bending deformation because there is no assembly procedure for this rotor.

4.1. Stability and Bifurcation of the Imprecise Bolt-Disc Rotor. The global stability boundaries and areas of imprecise boltdisc and monobloc rotor systems are demonstrated in Figure 6.

The global stability boundaries are determined by the eigenvalues (Floquet multipliers) of the Jacobian matrix $\partial \mathbf{H} /$ $\partial \mathbf{Y}$ in equation (7) in the paper [11-14]. For a stable periodic solution, all the Floquet multipliers should be within the unit circle in the complex plane. The largest magnitude among all eigenvalues of the matrix is called the leading Floquet multiplier. Generally, the common double-periodic will happen when a real leading Floquet multiplier crosses the unit circle on the negative real axis. In addition, the quasiperiodic bifurcation will happen when a pair of complex conjugate leading Floquet multipliers crosses the unit circle.

$B_{1}$ and $B_{2}$ stand for quasiperiodic (QP) and doubleperiodic (DP) solution boundaries for the imprecise boltdisc rotor system. $\mathrm{B}_{1}{ }_{1}$ and $\mathrm{B}_{2}{ }_{2}$ mean $\mathrm{QP}$ and $\mathrm{DP}$ boundaries for the monobloc rotor system. $\mathrm{P}$ represents periodic solution area in which the rotor system has stable periodic orbits. The global stability areas of bolt-disc and monobloc rotor systems are composed of three parts: P, QP, and DP area.

The curve $\left(e+r_{\mathrm{d}}\right)$ and line $(e)$ are extracted from the results of Figure 7 . Curve $\left(e+\mathbf{r}_{d}\right)$ and $\mathrm{B}_{1}$ have a crossing point $v$; line $(e)$ and $\mathrm{B}^{\prime}{ }_{1}$ have a crossing point $v^{\prime}$. If $\omega<\omega_{v}\left(\omega_{v^{\prime}}\right)$, the bolt-disc (monobloc) rotor will perform $\mathrm{P}$ motions; if $\omega>\omega_{v}\left(\omega_{v^{\prime}}\right)$, the bolt-disc (monobloc) rotor behave QP motions.

It can be observed that $\mathrm{P}$ area of bolt-disc rotor is obviously smaller than that of monobloc rotor. It means that rotor flexure due to position error of bolt-holes has the lower system stability. 


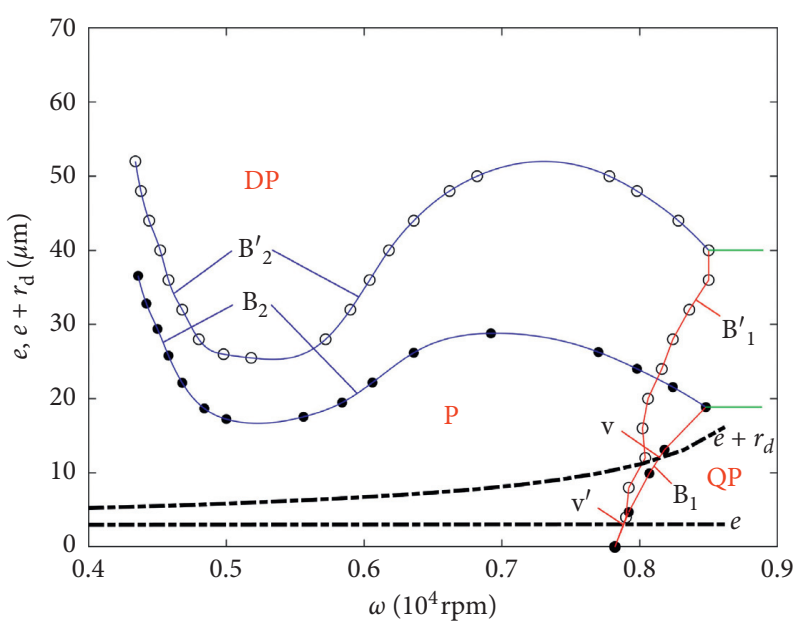

Bolt-disc rotor

- Monobloc rotor

FIGURE 6: Global stability boundaries and areas.

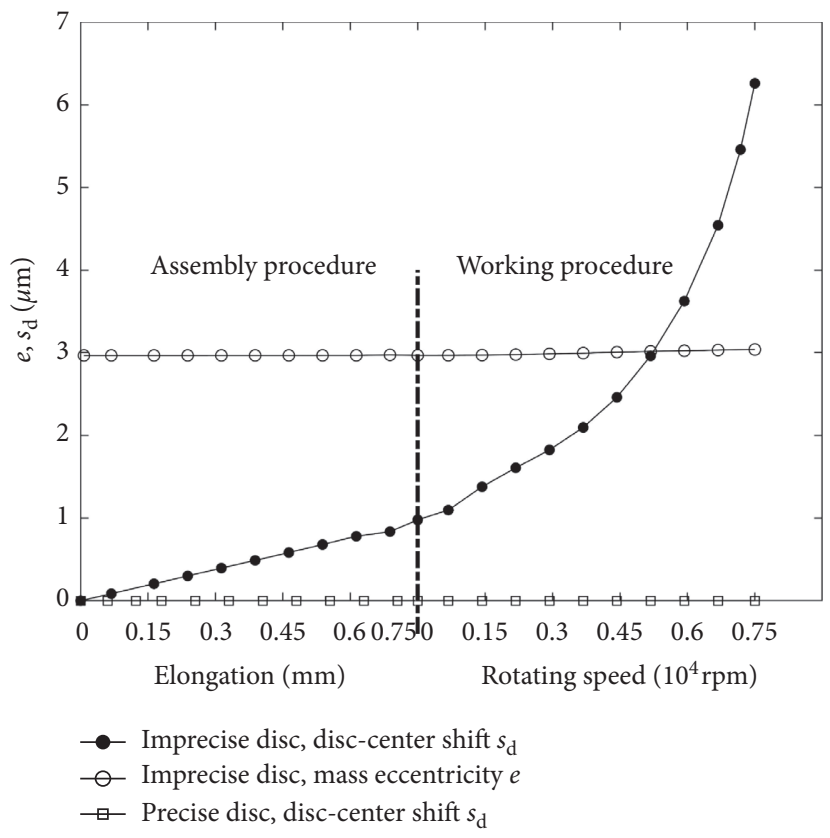

FIgURE 7: Axis-center shift and mass eccentricity and of disc "b" with the position error of bolt-holes.

4.2. Nonlinear Vibration. The vibration in Figure 8(a) indicates that both rotor systems almost have the same critical speed $\left(\omega_{\mathrm{c}} \approx 4800 \mathrm{rpm}\right)$, but its amplitude $A_{c}$ of bolt-disc rotor is larger than that of monobloc rotor. Thus, speed-variant rotor bending hardly influences critical speed, but it can bring about larger vibration.

There is a distinctive feature here for imprecise bolt-disc rotor. Before balance, the amplitude rises again when $\omega>\omega_{c}$; after balance (balance speed equals $\omega_{c}$ ), the vibration also grows and becomes larger than critical amplitude $A_{c}$ when $\omega>\omega_{c}$.

This performance is also found in the vibration response of gas turbine rotor (Figure 1) which is also a typical bolt- disc rotor. The data in Figure $8(\mathrm{~b})$ are measured in the dynamic balancing process which is not influenced by gas and heat. Displacement sensors are used to measure shaft vibration. At a certain speed, the rotor stays for several minutes to detect the vibration value. Similarly, no matter whether the F-class gas turbine rotor has dynamic balance (balance speed equals its second critical speed $2200 \mathrm{rpm}$ ), the vibration rises again after $\omega_{c}$.

The reason is clear when analyzing the vibration of imprecise bolt-disc rotor. Because the balance speed equals $\omega_{c}$ to reduce its excitation $\mathbf{F}_{c}$ as much as possible, the balance vector $\mathbf{B}_{c}$ has to meet the following relation: 


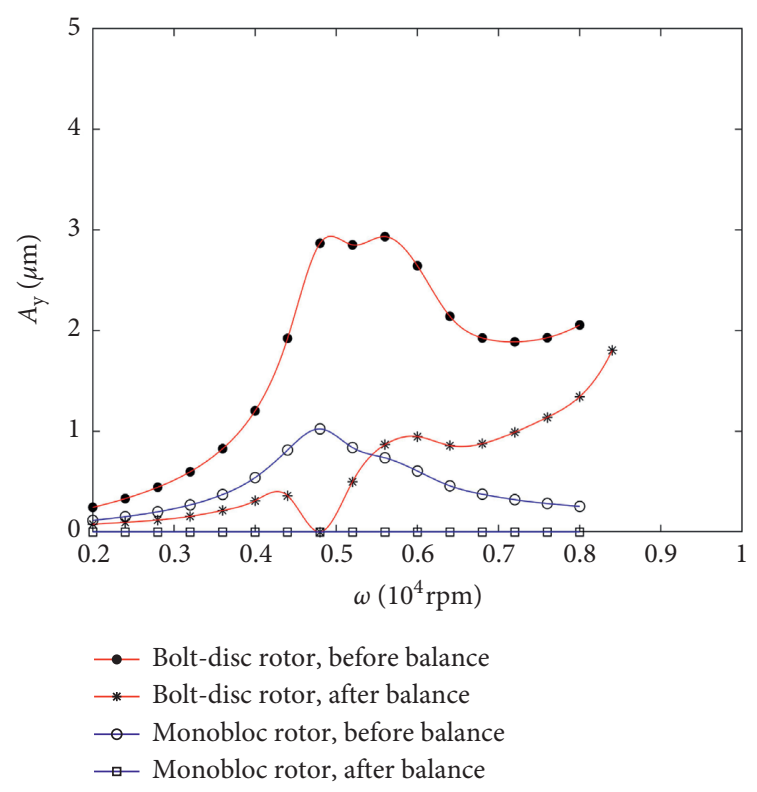

(a)

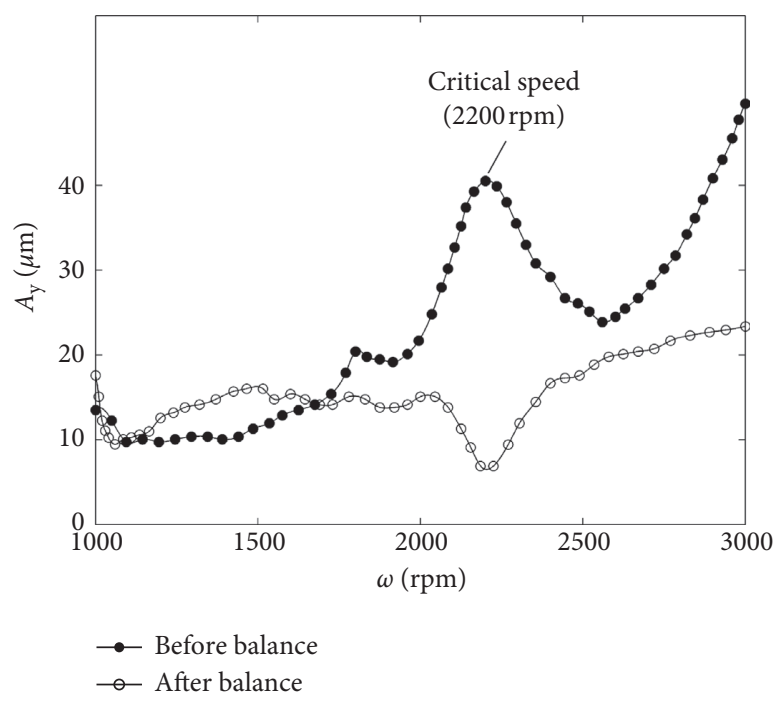

(b)

Figure 8: Vibration for bolt-disc rotor and F-class gas turbine rotor: (a) vibration for bolt-disc rotor and (b) vibration for the F-class gas turbine rotor.

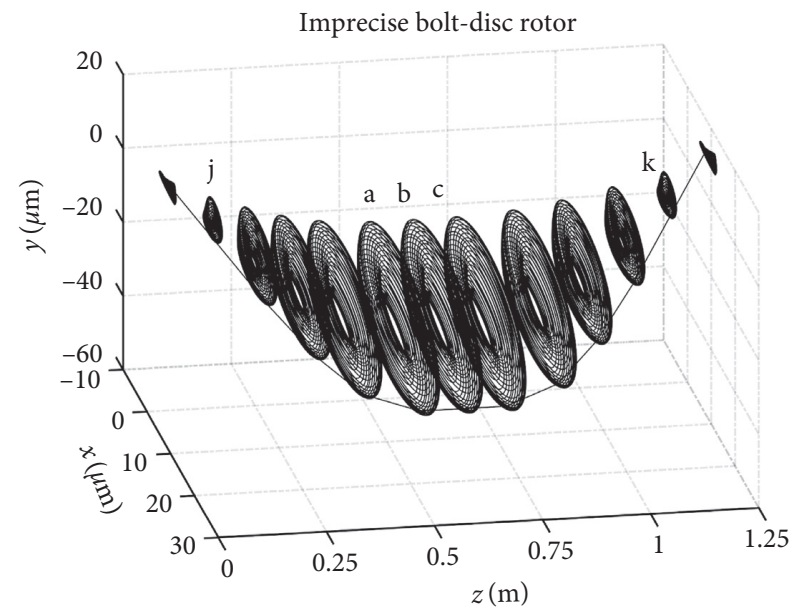

(a)

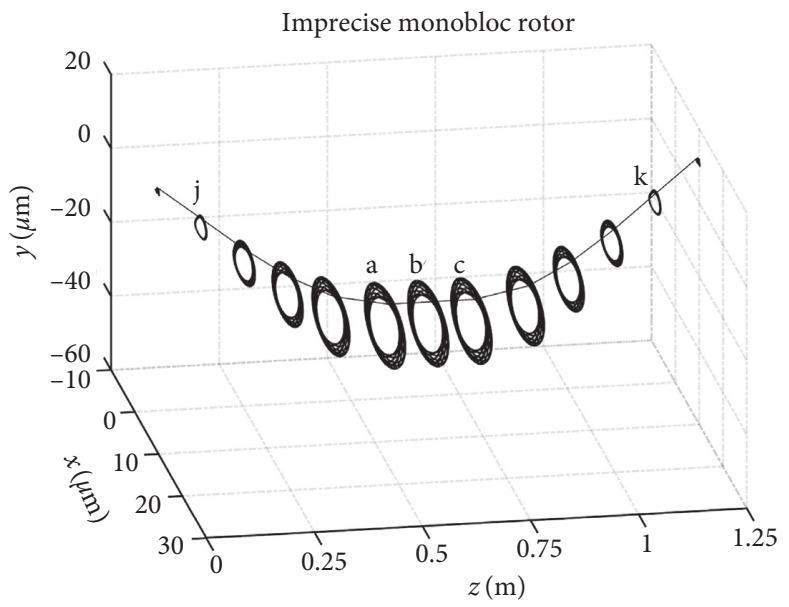

(b)

Figure 9: Dynamic motions for the both imprecise rotor: (a) quasiperiodic motion of bolt-disc rotor at $8170 \mathrm{rpm}$ and (b) quasiperiodic motion of monobloc rotor at $7850 \mathrm{rpm}$.

$$
\begin{aligned}
& \mathbf{B}_{c}=-\left(m \mathbf{e}+\frac{\mathbf{K}^{R} \mathbf{r}_{d}}{\omega_{c}^{2}}\right), \\
& \mathbf{F}_{c}=\left|\mathbf{B}_{c}+\left(m \mathbf{e}+\frac{\mathbf{K}^{R} \mathbf{r}_{d}}{\omega_{c}^{2}}\right)\right|=0 .
\end{aligned}
$$

When rotor has a balance vector $\mathbf{B}_{c}$ at critical speed, the vibration amplitude equals zero theoretically because the excitation is totally balanced at $\omega=\omega_{c}$. However, for other speeds $\left(\omega \neq \omega_{c}\right)$, the excitation vector $\mathbf{F}$ is rewritten as

$$
\mathbf{F}=\left|\mathbf{B}_{c}+\left(m \mathbf{e}+\frac{\mathbf{K}^{R} \mathbf{r}_{d}}{\omega^{2}}\right)\right|=\frac{\mathbf{K}^{R} \mathbf{r}_{d}}{\omega_{c}^{2} \omega^{2}}\left|\omega_{c}^{2}-\omega^{2}\right| .
$$

As a result, the excitation at other speeds is larger than $\mathbf{F}_{c}$. In addition, $\mathbf{F}$ becomes larger as speed increases when $\omega>\omega_{c}$. Therefore, the vibration amplitude rises again after critical speed.

This special phenomenon gives the reason why the position error of bolt-holes in the rotor is highly demanded. It also indicates this machining error should be examined carefully when designing this kind of combined rotor. 


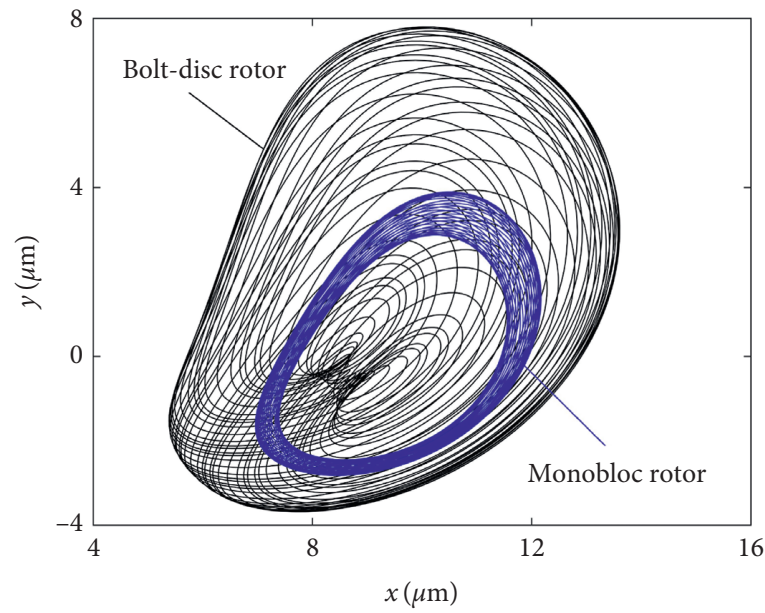

(a)

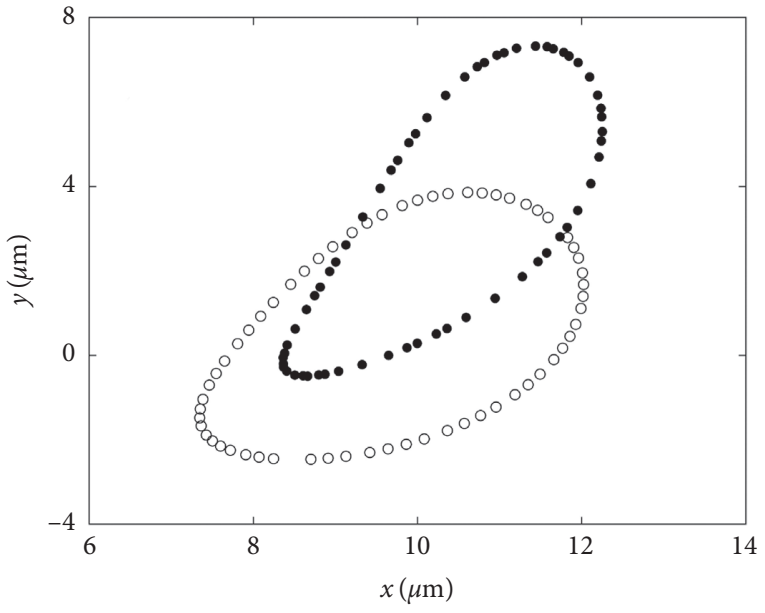

- Bolt-disc rotor

- Monobloc rotor

(b)

Figure 10: Whirling motions of the both imprecise rotors with position error at the bearing " $k$ ": (a) rotor orbits at bearing " $k$ " and (b) correspondent Poincaré map.

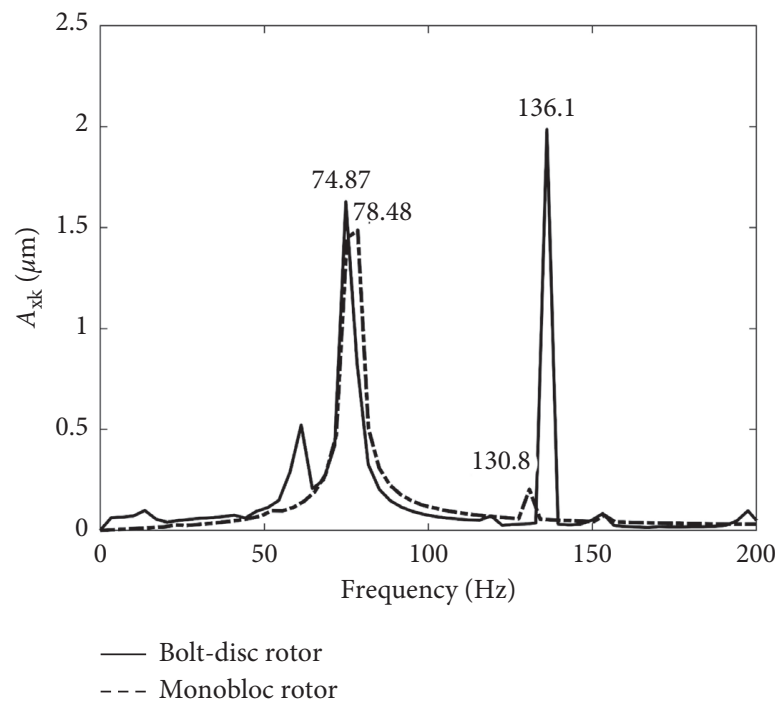

Figure 11: Frequency spectrums of both imprecise rotors with position error at the bearing "k."

\subsection{Nonlinear Vibration Characteristics of Imprecise Rotors.} Figure 6 shows that the imprecise bolt-disc and monobloc rotor bearing systems lose local stabilities at $8170 \mathrm{rpm}$ and $7850 \mathrm{rpm}$. In Figures 9(a) and 9(b), the quasiperiodic motions of 13 nodes on the rotor axis are shown and the line connecting the orbits is the transient vibration mode of the deformed rotor.

Figure 10(a) compares the whirling orbits of the both imprecise rotors at the node of bearing "k." Figure 10(b) reveals that the motions of imprecise bolt-disc rotor and imprecise monobloc rotor are typically quasiperiodic because the corresponding Poincaré points form a closed curve.
The fast Fourier transform (FFT) is performed to obtain the frequency spectrums of the imprecise rotors. Figure 11 shows that both systems have the similar frequency features: harmonic frequency, oil-film instability frequency, and high frequencies.

Harmonic frequency $(136.1 \mathrm{~Hz}$ and $130.8 \mathrm{~Hz})$ is calculated according to the rotating speed; oil-film instability frequency $(74.87 \mathrm{~Hz}$ and $78.48 \mathrm{~Hz}$ ) approximately equals the half of harmonic frequency which originates from the Hopf periodic solution of the balanced rotor system and the effect of oil-film oscillation occurs to the rotor; the high frequencies stem from the quasiperiodic motion. 


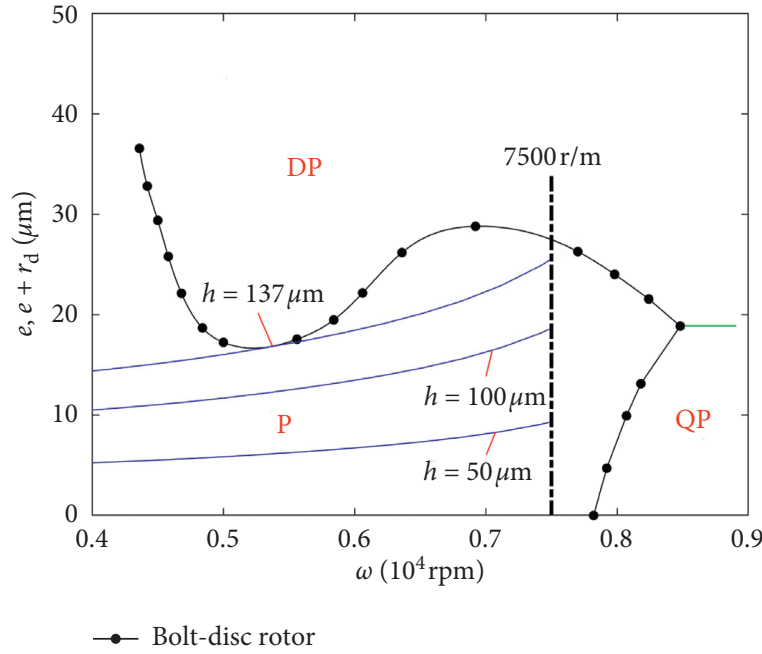

(a)

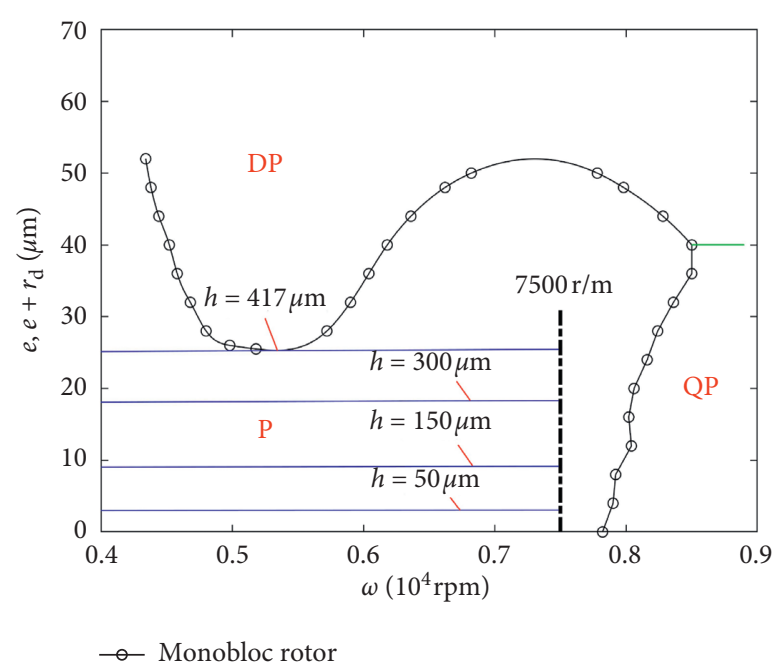

(b)

Figure 12: Allowable position error for both rotors: (a) allowable position error for bolt-disc rotor and (b) allowable position error for monobloc rotor.

4.4. Allowable Position Error. There is an unchanged mass eccentricity $e$ and speed-variant rotor bending $\mathbf{r}_{d}$ in the bolt-disc rotor when the position error of bolt-holes exists among discs. Excessive $e$ and $\mathbf{r}_{d}$ will bring bad effects on the dynamic characteristics. For the sake of controlling these factors effectively, the maximum allowable error should be ensured.

For the stability diagram (Figure 12) of imprecise boltdisc rotor and monobloc rotor, curve $\left(e+\mathbf{r}_{d}\right)$ and line $(e)$ gradually approach their stability boundaries when $e$ and $\mathbf{r}_{d}$ increase from 0 to working speed $(7500 \mathrm{rpm})$. In this speed range, both rotors should work in the stable area (P area) all the time. So, the maximum allowable position error can be found out when curve $\left(e+\mathbf{r}_{d}\right)$ and line (e) are tangent to boundary $\mathrm{B}_{2}$ and $\mathrm{B}_{2}$, respectively.

The analysis results show that the maximum allowable position error of bolt-holes for bolt-disc rotor and monobloc rotor is $137 \mu \mathrm{m}$ and $417 \mu \mathrm{m}$. This tolerance also indicates a reference of bolt position precision when designing this combined rotor.

\section{Conclusions}

This work presents a static-dynamic uniting analysis to study the influences of position error of bolt-holes in a typical boltdisc rotor system. The conclusions include the following:

(1) The deviation of position error of bolt-holes causes mass eccentricity and rotor bending due to tightening elongation and working speed for the imprecise disc.

(2) The unbalance component including mass eccentricity and rotor bending originating from position error of bolt-holes leads to obvious stability reduction and vibration growth.

(3) The vibration amplitude of imprecise bolt-disc rotor rises again when $\omega>\omega_{c}$ before (or after) dynamic balance. This property also exists in an F-class gas turbine rotor and is obviously different from monobloc rotor.

(4) The allowable position error of bolt-holes is obviously smaller than that of monobloc rotor. It shows that this assembly precision should be restricted to avoid position error as much as possible.

\section{Data Availability}

The data used to support the findings of this study are included within the article.

\section{Conflicts of Interest}

The authors declare that they have no conflicts of interest about this paper.

\section{Acknowledgments}

The work described in this paper was funded by a grant from the National Science Funds of China (no. 51705399) and Aeronautics Power Foundation (no. 6141B090547).

\section{References}

[1] K.-B. Lee, Y.-B. Kim, and K.-Y. Jeong, "Identification of rotor bearing parameter using orbit data," Proceedings of the Institution of Mechanical Engineers, Part K: Journal of MultiBody Dynamics, vol. 219, no. 3, pp. 249-257, 2005.

[2] Y. J. Lu, R. Dai, D. Hei, Y. F. Zhang, H. Liu, and L. Yu, "Stability and bifurcation of a non-linear bearing-flexible rotor coupling dynamic system," Proceedings of the Institution of Mechanical Engineers, Part C: Journal of Mechanical Engineering Science, vol. 223, no. 4, pp. 835-849, 2009.

[3] M. Cheng, G. Meng, and J. P. Jing, "Numerical study of a rotor-bearing-seal system," Proceedings of the Institution of 
Mechanical Engineers, Part C: Journal of Mechanical Engineering Science, vol. 221, no. 7, pp. 779-788, 2007.

[4] J. Yi, H. Liu, F. T. Wang, M. Li, and M. Jing, "The resonance effect induced by the variable compliance vibration for an elastic rotor supported by roller bearings," Proceedings of the Institution of Mechanical Engineers, Part C: J. Multi-Body Dynamics, vol. 228, no. 4, pp. 779-788, 2014.

[5] Z. Wan, J.-P. Jing, G. Meng, Y. Yang, and H.-Y. Bai, "Theoretical and experimental study on the dynamic response of multi-disk rotor system with flexible coupling misalignment," Proceedings of the Institution of Mechanical Engineers, Part C: Journal of Mechanical Engineering Science, vol. 226, no. 12, pp. 2874-2886, 2012.

[6] Q. Yuan, J. Gao, and P. Li, "Nonlinear dynamics of the rodfastened Jeffcott rotor," ASME Journal of Applied Mechanics, vol. 136, p. 021011, 2014.

[7] Y. Liu, H. Liu, J. Yi, and M. Q. Jing, "Investigation on the stability and bifurcation of a 3D rotor-bearing system," ASME Journal of Vibration and Acoustics, vol. 135, p. 031017, 2013.

[8] Y. Liu, H. Liu, X. Wang, and M. Jing, "Nonlinear dynamic characteristics of a three-dimensional rod-fastening rotor bearing system," Proceedings of the Institution of Mechanical Engineers, Part C: Journal of Mechanical Engineering Science, vol. 229, no. 5, pp. 882-894, 2015.

[9] K. J. Bathe, Finite Element Procedures, Prentice-Hall, Hoboken, NJ, USA, 1996.

[10] P. Wriggers, "Finite element algorithms for contact problems," Archives of Computational Methods in Engineering, vol. 2, no. 4, pp. 1-49, 1995.

[11] P. Sundararajan and S. T. Noah, "Dynamics of forced nonlinear systems using shooting/arc-length continuation method-application to rotor systems," Journal of Vibration and Acoustics, vol. 119, no. 1, pp. 9-20, 1997.

[12] R. H. B. Fey, D. H. Van Campen, and A. de Kraker, "Long term structural dynamics of mechanical systems with local nonlinearities," Journal of Vibration and Acoustics, vol. 118, no. 2, pp. 147-153, 1996.

[13] T. S. Parker and L. O. Chua, Practical Numerical Algorithms for Chaotic System, Springer-Verlag, New York, NY, USA, 1989.

[14] R. Seydel, From Equilibrium to Chaos, Practical Bifurcation and Stability Analysis, Elsevier, New York, NY, USA, 1988.

[15] O. Pinkus and B. Sternlicit, Theory of Hydro-Dynamic Lubrication, McGraw-Hill, New York, NY, USA, 1961.

[16] E. L. B. Van De Vorst, R. H. B. Fey, A. De Kraker, and D. H. Van Campen, "Steady-state behaviour of flexible rotordynamic systems with oil journal bearings," Nonlinear Dynamics, vol. 11, no. 3, pp. 295-313, 1996. 